\title{
La diffusion quasiélastique cohérente des neutrons
}

\author{
G. Coddens
}

Laboratoire Léon Brillouin, CEA-CNRS, 91191 Gif-sur-Yvette cedex, France

\begin{abstract}
Résumé. Nous expliquons la différence entre la diffusion cohérente et la diffusion incohérente des neutrons par une analogie avec l'expérience de diffraction par une double fente de Young. Nous montrons que le langage adéquat pour calculer les signaux cohérents est basé sur une description en termes de configurations dans lesquelles les particules identiques qui peuvent être échangées sont considérées comme indiscernables. Nous illustrons notre démarche avec un exemple tiré de la physique des quasicristaux.
\end{abstract}

\section{Introduction}

Le formalisme de Van Hove [1] est à la base de tout développement théorique en diffusion neutronique. Il permet d'exprimer les sections efficaces différentielles de diffusion cohérente et in- cohérente sous la forme:

$$
\begin{aligned}
& {\left[\frac{d^{2} \sigma}{d \Omega d E_{f}}\right]_{i n c}=\frac{k_{f}}{k_{i}} \frac{1}{2 \pi \hbar} \int_{-\infty}^{\infty} e^{-w \omega t} \sum_{j}\left[b_{j}^{(i n c)}\right]^{*}\left[b_{j}^{(i n c)}\right]\left\langle e^{\imath \boldsymbol{Q} \cdot\left(\boldsymbol{r}_{j}(t)-\boldsymbol{r}_{j}(0)\right)}\right\rangle_{t h} d t} \\
& {\left[\frac{d^{2} \sigma}{d \Omega d E_{f}}\right]_{c o h}=\frac{k_{f}}{k_{i}} \frac{1}{2 \pi \hbar} \int_{-\infty}^{\infty} e^{-w \omega t} \sum_{j, k}\left[b_{j}^{(\text {coh })}\right]^{*}\left[b_{k}^{(\text {coh })}\right]\left\langle e^{\imath \boldsymbol{Q} \cdot\left(\boldsymbol{r}_{k}(t)-\boldsymbol{r}_{j}(0)\right)}\right\rangle_{t h} d t}
\end{aligned}
$$

Ici $b_{j}^{(i n c)}$ et $b_{j}^{(\text {coh })}$ sont les longueurs de diffusion incohérente et cohérente du noyau $j$. Les indices $j$ et $k$ parcourent tous les noyaux du système physique considéré. Ces noyaux se trouvent à l'endroit $\boldsymbol{r}_{j}(t)$ à l'instant $t ; \hbar \boldsymbol{k}_{i}, \hbar \boldsymbol{k}_{f}$ sont les moments initial et final du neutron diffusé et $\boldsymbol{Q}=\boldsymbol{k}_{\boldsymbol{f}}-\boldsymbol{k}_{i}$ le transfert de moment; de façon analogue $\hbar \omega=E_{f}-E_{i}$ est le transfert d'énergie. Finalement, $\langle\cdot\rangle_{t h}$ dénote une moyenne thermique. Dans la suite nous avons omis les indices coh et inc pour les longueurs de diffusion, la notation étant évidente dans le contexte. Les fonctions de diffusion incohérente et totale sont définies comme:

$$
\begin{aligned}
S_{i n c}(\boldsymbol{Q}, \omega) & \left.=\frac{1}{2 \pi} \int_{-\infty}^{\infty} e^{-\imath \omega t} \sum_{j}<e^{\imath \boldsymbol{Q} \cdot\left(\boldsymbol{r}_{j}(t)-\boldsymbol{r}_{j}(0)\right)}\right\rangle_{t h} d t=\frac{1}{2 \pi} \int_{-\infty}^{\infty} e^{-\imath \omega t} S_{i n c}(\boldsymbol{Q}, t) d t \\
S(\boldsymbol{Q}, \omega) & =\frac{1}{2 \pi} \int_{-\infty}^{\infty} e^{-\imath \omega t} \sum_{j, k}<e^{\imath \boldsymbol{Q} \cdot\left(\boldsymbol{r}_{k}(t)-\boldsymbol{r}_{j}(0)\right)}>_{t h} d t=\frac{1}{2 \pi} \int_{-\infty}^{\infty} e^{-\imath \omega t} S(\boldsymbol{Q}, t) d t
\end{aligned}
$$

Ce sont les transformées spatio-temporelles des fonctions de corrélation de Van Hove, qui expriment la probabilité de trouver une particule à l'endroit $r$ à l'instant $t$ lorsqu'une particule est présente à 
l'endroit 0 à l'instant 0 : Dans le cas de la diffusion incohérente, il s'agit de la même particule aux deux instants. Dans le cas de la diffusion cohérente cette restriction tombe. C'est pour cette raison que dans ce dernier cas la somme contient aussi des termes $j \neq k$. L'équation (1) peut s'obtenir à partir de l'équation (2) en y ajoutant le symbole de Kronecker $\delta_{j k}$. Ceci nous permet d'apprécier une chose importante: au moins dans le principe la compréhension de la diffusion cohérente n'est pas plus compliquée que celle de la diffusion incohérente. En effet, les sommes dans les équations (1) et (2) portent sur les mêmes quantités. On est amené à résoudre le même problème de probabilités dans les deux cas. L'unique différence réside dans la présence ou l'absence du symbole de Kronecker. L'usage veut que la fonction de diffusion correspondant à la diffusion cohérente soit appelée totale et écrite sans indice coh comme on aurait pu le prévoir. Ceci traduit que l'expression (4) pour la diffusion cohérente contient aussi les autocorrélations, c.à.d. les termes $j=k$. Cette partie "self" peut d'ailleurs être isolée aux grandes valeurs de $Q$, où la diffusion cohérente se comporte comme la diffusion incohérente. (Mais elle reste pondérée par les sections efficaces cohérentes des noyaux).

Le modèle type de diffusion quasiélastique incohérente est celui [2] d'une particule effectuant des sauts stochastiques entre deux sites equivalents séparés par un vecteur $\boldsymbol{d}$, avec un temps de relaxation $\tau$. Sa solution est simple et consiste à résoudre une équation différentielle linéaire du premier ordre basée sur une matrice de saut. Cette solution est une fonction de corrélation de Van Hove dont il convient de faire ensuite la transformée de Fourier spatio-temporelle. On s'attend à ce qu'une procédure analogue s'applique dans le cas de la diffusion cohérente, où la difficulté semble être accrue par le fait qu'il faut suivre l'évolution simultanée de toutes les particules. Ceci peut être compliqué par la présence de corrélations: une particule ne pourra sauter que quand une autre se sera déplac; 'ee de telle manière à ce que l'environnement soit propice au saut. Dans ces conditions il devient même difficile de mettre le problème en équation. Une astuce purement mathématique permet de prendre en compte l'influence possible de l'environnement; elle consiste à formuler le problème en termes de configurations. A priori, il faudra individualiser chaque particule pour décrire l'information complète du système. Quand on augmente le nombre $n$ de particules dans le système, le nombre de configurations peut devenir gigantesque. Dans certains cas, ce nombre croit comme $n$ ! Nous verrons que d'une certaine façon, la mécanique quantique nous sauve ici la vie en réduisant considérablement le nombre de configurations qu'il faut prendre en compte. C'est un point essentiel à retenir de ce chapitre. Nous pouvons donner une idée de la réduction de la complexité qui en résulte. Prenons par exemple le jeu de pousse-pousse inventé par Sam Lloyd au XIXième siècle. Nous avons certainement joué avec ce jeu pendant notre enfance. C'est un petit carré de dimensions $4 \times 4$ qui contient 15 pièces carrées, numérotées de 1 à 15 et un trou. Le jeu consiste à glisser les pièces afin de rétablir la situation d'origine où elles se trouvaient toutes en ordre croissant avec le trou en position ultime. Si on considère les pièces numérotées comme des particules identiques, il n'y aura que 16 configurations à observer pour un calcul de diffusion cohérente. Celles-ci seront entièrement définies par la position du trou. Si on veut faire un calcul de diffusion incohérente, il faudra également savoir 
où se trouvera une pièce choisie. Il y aura alors $16 \times 15$ configurations à considérer. Si on avait été obligé de suivre les positions simultanées de toutes les pièces, ce nombre aurait été beaucoup plus grand (il est de l'ordre de $10^{13}$ ). Il y a des corrélations entre les positions des différentes pièces de sorte que l'on n'explore que la moitié des 16! permutations imaginables [3]. Mais on peut proposer une variante du jeu pour laquelle toutes les 16 ! possibilités seraient réalisées. Pour cela il suffira de définir le mouvement élémentaire du jeu comme le saut d'une pièce quelconque vers le trou.

\section{Rappel sur la diffusion cohérente et incohérente}

Il est intéressant de vérifier la définition des sections efficaces de diffusion cohérente et incohérente d'un noyau dans un manuel de physique nucléaire. On y explique que la section efficace de diffusion incohérente implique une inversion du spin du neutron, tandis que la diffusion cohérente correspond à un processus sans changement de spin. Il s'agit donc d'une propriété d'un noyau correspondant à une réaction nucléaire bien précise, définie pour un seul noyau. Dans la terminologie des physiciens nucléaires, la diffusion en question est d'ailleurs qualifiée d'élastique puisqu'elle n'implique pas une excitation du noyau vers un autre niveau.

Cependant, les manuels de physique de la matière condensée nous tiennent un propos différent. Ils associent la diffusion cohérente à une information collective et la diffusion incohérente à un comportement individuel. Quel est le lien avec les énoncés des physiciens nucléaires? Comment la réaction nucléaire peut-elle nous faire parvenir une information collective sur plusieurs particules?

La "réponse" à ces questions est donnée par Richard Feynman dans son cours sur la mécanique quantique [4]. Il montre comment des questions analogues se posent pour l'expérience de Young relative à la diffraction d'un faisceau de particules par une double fente. Un premier paradoxe dans cette expérience, est que d'une part l'intensité est typique d'un phènomène ondulatoire (présentant de fortes oscillations par les effets d'interférence) et que d'autre part ce sont bien des particules qui arrivent dans le dispositif de détection. Ces particules arrivent une par une. L'intensité mesurée est quantisée. Ce n'est jamais une fraction d'une particule qui arrive. Au début on ne voit pas les franges d'interférences et il est difficile de discerner si le profil d'intensité correspond à celui d'une double fente ou à une superposition de deux profils de fentes uniques. C'est seulement après l'enrégistrement de milliers de particules que l'image des franges d'interférences commence à se dessiner clairement. Cette évolution temporelle du diagramme de diffraction a été merveilleusement illustrée dans la référence [5]. Nous incitons nos lecteurs à consulter cette référence pour en examiner les photos afin de voir comment le diagramme de diffraction se construit progressivement en fonction du temps d'exposition de la plaque photographique. Quand on essaie de déterminer par laquelle des deux fentes les particules passent, les franges d'interférences et le comportement ondulatoire disparaissent faisant place à une superposition de deux distributions d'intensité classiques caractéristiques du comportement d'une 
particule dans une expérience avec une seule fente. On pourrait par exemple s'imaginer que l'on essaie de rendre la particule visible en l'illuminant avec une torche. Quand la longueur d'onde de la lumière envoyée devient assez petite pour définir la place de la particule avec une bonne précision, l'énergie des photons devient suffisamment grande pour qu'elle affecte la trajectoire de la particule et les franges d'interférence commencent à disparaitre. Aussi, quand on bouche un des deux trous, on saura parfaitement que la particule sera passée par l'autre trou mais on retrouve une distribution d'intensité classique pour une seule fente. En résumé, quand on arrive à collecter des informations qui permettent de retracer la trajectoire de la particule, on trouve un comportement classique. Si l'on s'en abstient, on trouve un comportement ondulatoire. Cette règle énoncée s'avère universellement vérifiée. La nature se comporte de ce point de vue comme une machinerie parfaite. Feynman nous explique que cette expérience contient l'unique mystère de la mécanique quantique et pose la question qui soulève le second paradoxe présent dans cette expérience: comment la particule qui passe par une fente peut savoir si l'autre fente est ouverte ou non? Le fait que la nature puisse respecter toujours la règle énoncée paraît effectivement miraculeux. Personne n'est arrivé à l'expliquer en proposant un mécanisme microscopique détaillé.

La diffusion cohérente des neutrons correspond de façon analogue à un comportement ondulatoire, tandis que dans la diffusion incohérente, le neutron se comporte comme une particule. En effet, considérons un réseau de noyaux indentiques aux spins tous parallèles et supposons pour simplifier que ces noyaux ont un spin $\frac{1}{2}$. Ceci nous permettra de nous affranchir de certains détails encombrants mais inessentiels. La diffusion cohérente d'un neutron, par un noyau d'un tel réseau, nous rendra exactement la même "photo" avant et après le processus de diffusion puisqu'elle n'inverse pas l'orientation du spin du neutron. La conservation du moment angulaire nous permet alors d'affirmer qu'elle n'affecte pas le spin du noyau non plus. On ne pourra donc pas dire après un examen aussi méticuleux qu'il soit, lequel de ces noyaux aurait diffusé le neutron. En analogie avec l'expérience de la double fente, on ne sait pas reconstituer la trajectoire du neutron, puisqu'aucun noyau a enrégistré un indice de son passage. Ceci correspond à un comportement ondulatoire et donne donc une information sur une collectivité d'atomes. Cette information est caractérisée par de fortes oscillations d'intensité relévées par les effets d'interférences (par exemple les pics de Bragg). Par contre, dans le cas d'une diffusion incohérente le spin d'un noyau aura été affecté et ce noyau s'en trouvera marqué ou, si on veut, pourvu d'une étiquette. On pourra donc reconstituer la trajectoire du neutron. On retouve alors un comportement de particule et l'information est obtenue à l'échelle de l'atome individuel. La distribution d'intensité mesurée sera caractérisée par une variation lisse sans fortes oscillations. L'opposition entre la diffusion cohérente et la diffusion incohérente des neutrons n'est donc rien d'autre que la dualité onde-particule. Le lecteur attentif aura remarqué que nous n'avons pas vraiment répondu aux questions évoquées plus haut. La bonne raison est que nous ne connaissons pas cette réponse. C'est pourquoi le mot "réponse" doit rester entre guillemets. Nous ignorons parfaitement quel est le mécanisme qui permet de produire les intensités observées, mais 
nous savons que cette incompréhension est la même que celle que l'on rencontre partout dans la mécanique quantique. C'est celle qui opère dans l'expérience de la double fente et nous savons qu'elle est l'unique mystère de la mécanique quantique.

\section{Idée de base de la méthode}

Ces remarques sont à la base de notre méthode pour décrire la diffusion quasiélastique cohérente. Imaginons par exemple dans une expérience de pensée que l'on échange deux noyaux identiques par diffusion cohérente d'un neutron mais que pour le reste il ne se passe rien de sorte que les photos avant et aprés diffusion soient identiques. C'est comme si rien ne s'était passé et la réponse neutronique va nous en fournir une information conséquente. Il s'ensuit que ce qui est important pour la diffusion cohérente est uniquement la configuration des noyaux. L'information sur l'identité des atomes individuels dans cette configuration n'est pas accessible. Il faut donc également décrire les données uniquement en termes d'informations disponibles, puisque les considérations, sur ce qui n'est pas accessible, ne peuvent que compliquer notre entreprise. Par une voie complètement indépendante, nous retrouvons donc que les configurations sont importantes pour la diffusion cohérente des neutrons. Les deux raisonnements font appel à des idées très différentes, l'une plutôt physique, l'autre plutôt mathématique. Mais la physique nous dit quelque chose de plus: c'est que nous n'avons pas besoin de marquer les particules identiques qui peuvent être réellement échangées entre elles [6]. On peut les considérer comme indiscernables. Ceci entrainera une grande économie dans le nombre de configurations à considérer. Cela n’implique pas que ces particules soient quantiques. Elles peuvent être parfaitement classiques. L'indiscernabilité provient du comportement quantique du neutron. Nous allons maintenant développer ces idées.

Nous écrivons la fonction de diffusion totale qui correspond à la réponse du sytème pour la diffusion cohérente:

$$
S(\boldsymbol{Q}, \omega)=\frac{1}{2 \pi} \int_{-\infty}^{\infty} e^{-\imath \omega t} \sum_{j, k} b_{j}^{*} b_{k}<e^{\imath \boldsymbol{Q} \cdot\left(\boldsymbol{r}_{k}(t)-\boldsymbol{r}_{j}(0)\right)}>_{t h} d t
$$

où par abus de langage nous avons inclus les longueurs de diffusion cohérente dans la définition de $S$. Ceci ne posera aucun problème si tous les atomes sont du même type (isotopique). Introduisons maintenant les quantités

$$
\mathcal{F}(\boldsymbol{Q}, t)=\sum_{k} b_{k} e^{\imath \boldsymbol{Q} \cdot \boldsymbol{r}_{k}(t)}=\frac{1}{(2 \pi)^{3}} \int_{\mathbb{R}^{3}} e^{\imath \boldsymbol{Q} \cdot \boldsymbol{r}} \sum_{k} b_{k} \cdot \delta\left(\boldsymbol{r}-\boldsymbol{r}_{k}\right) d \boldsymbol{r}
$$


Alors

$$
\sum_{j, k} b_{j}^{*} b_{k}<e^{\imath \boldsymbol{Q} \cdot\left(\boldsymbol{r}_{k}(t)-\boldsymbol{r}_{j}(0)\right)}>_{t h}=\left\langle\mathcal{F}(\boldsymbol{Q}, t) \mathcal{F}^{*}(\boldsymbol{Q}, 0)>_{t h}\right.
$$

où $\mathcal{F}(\boldsymbol{Q}, t)$ n'est rien d'autre que la transformée de Fourier spatiale d'une configuration de mesures de Dirac de poids $b_{k}$ placées aux positions $\boldsymbol{r}_{k}$. Cette transformée de Fourier ne changera pas lors d'un échange de deux particules identiques comme expliqué avec les mains plus haut. Remarquons également que nous n'avons pas changé l'ordre des opérateurs dans les expressions: l'opérateur au moment $t$ précède toujours celui au moment 0 . Il est intéressant de vérifier le développement théorique du formalisme de diffusion quasiélastique des neutrons dans la monographie de Bée. L'équation (2.31) de ce livre correspond à l'équation (8) ci-dessous. L'équation (2.40) de Bée correspond à l'équation (7) et l'équation (2.41) à l'équation (1). Nous voyons que d'une certaine manière le développement proposé par Bée, bien que plus complet a conduit à une expression beaucoup moins utile, mais mathématiquement plus explicite. Nous avons donc:

$$
S(\boldsymbol{Q}, \omega)=\frac{1}{2 \pi} \int_{-\infty}^{\infty} e^{-\imath \omega t}<\mathcal{F}(\boldsymbol{Q}, t) \mathcal{F}^{*}(\boldsymbol{Q}, 0)>_{\text {th }} d t
$$

La méthode est donc complètement analogue à celle expliquée par Bée pour la diffusion incohérente. L'unique différence réside dans le fait que nous décrivons le système en termes de configurations au lieu de sites. Il convient donc de résoudre l'équation séculaire de la matrice de saut pour les configurations. Nous avons ainsi mis en place un isomorphisme mathématique. Nous pouvons nous imaginer que le système entier est une particule abstraite qui effectue des sauts de site abstrait en site abstrait. Les sites abstraits sont des configurations. Pour dénombrer ces configurations, il faut supposer que les permutations entre particules identiques pouvant être réellement échangées ne changent pas la configuration [7]. Au lieu de développer ce formalisme dans toute sa généralité mieux vaut en faire passer les idées sous-jacentes en travaillant sur un exemple. Notons que la généralisation implique l'introduction des fonctions $S^{\alpha \beta}(\boldsymbol{Q}, \omega)$ telles qu'elles sont définies par Bée.

\section{Développement de l'idée: un exemple de physique des quasicristaux}

La Figure 1 montre le système modèle dont nous voulons calculer la fonction de diffusion totale. C'est un morceau d'un pavage quasipériodique octogonal de type Penrose. Ce genre de pavages quasipériodiques servent de paradigme pour la structure des quasicristaux. Le morceau en question 

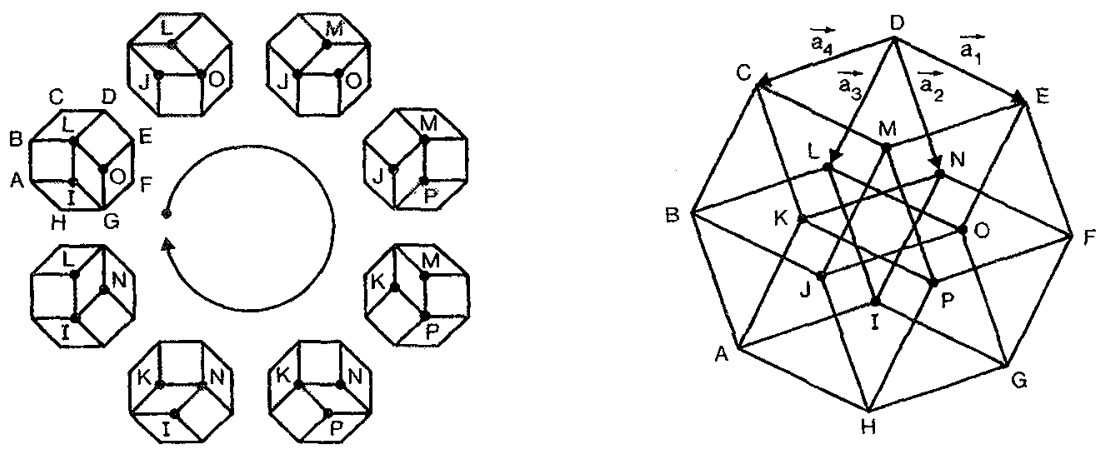

Figure 1: Morceau d'un pavage de Penrose octogonal. A droite, nous montrons l'ensemble des sites possibles sur la représentation de la projection de l'hypercube de $\mathbb{R}^{4}$ sur $\mathbb{R}^{2}$ (réf. [10]).

contient 11 atomes, dont huit sur le contour de l'octogone ABCDEFGH et trois à l'intérieur sur les sites I, L et $\mathrm{O}$. Le modèle de sauts que nous allons discuter a été introduit par Kalugin et Katz [8] qui l'ont proposé comme un mécanisme possible pour la diffusion atomique à grande distance dans les quasicristaux. Les sites BLOGHAI forment la projection bidimensionnelle d'un cube (imaginaire) vu en perspective.

Ceci est intimement lié au fait que ces pavages de Penrose sont obtenus par une méthode de projection d'un réseau périodique dans un espace de dimension supérieure. En changeant de perspective dans le cube imaginaire nous faisons sauter l'atome en I vers le site $\mathbf{J}$. A l'issue de cette opération, $\mathrm{L}$ se trouve à son tour dans une situation analogue à l'intérieur du cube virtuel donné par OJBCDEL. Remarquons que le saut de l'atome de $\mathrm{L}$ vers $\mathrm{M}$ n'était pas possible dans la situation de départ. Nous avons donc une dynamique complexe où l'occurrence de certains sauts peut dépendre d'autres sauts qui doivent être effectués au préalable. Décrire cette dynamique conditionnelle par une équation différentielle en termes de particules peut s'avérer très difficile. Par contre, comme le montre la figure 1, la description devient très simple en termes de configurations. Si nous ne faisons pas la distinction entre les particules, ce qui suffit pour une description du signal cohérent, nous n'avons à considérer que huit configurations. Après huit sauts nous avons fait le tour de l'espace des configurations et nous revenons à la situation de départ. Cependant les trois particules en $O, L$ et I auront subis une permutation cyclique. Notre particule abstraite diffuse alors par sauts sur un octogone régulier dont 
tous les sites sont équivalents. Ce problème de diffusion a été résolu dans le livre de Bée. L'évolution des probabilités est régie par l'équation séculaire:

$$
\frac{d}{d t} \mathbf{P}=\frac{1}{\tau} \mathbf{M P}
$$

où la matrice de saut $\mathbf{M}$ est donnée par:

$$
\mathbf{M}=\left(\begin{array}{cccccccc}
-2 & 1 & 0 & 0 & 0 & 0 & 0 & 1 \\
1 & -2 & 1 & 0 & 0 & 0 & 0 & 0 \\
0 & 1 & -2 & 1 & 0 & 0 & 0 & 0 \\
0 & 0 & 1 & -2 & 1 & 0 & 0 & 0 \\
0 & 0 & 0 & 1 & -2 & 1 & 0 & 0 \\
0 & 0 & 0 & 0 & 1 & -2 & 1 & 0 \\
0 & 0 & 0 & 0 & 0 & 1 & -2 & 1 \\
1 & 0 & 0 & 0 & 0 & 0 & 1 & -2
\end{array}\right)
$$

P est une matrice colonne $1 \times 8$ dont les éléments $p_{j}(t)$ donnent la probabilité de trouver la particule abstraite dans le site $j$ à l'instant $t$. Autrement dit, $p_{j}(t)$ est la probabilité de retrouver le système dans la configuration $j$ à l'instant $t$. La procédure utilisée pour résoudre une équation linéaire du style (9) est bien connue. Elle implique le calcul de la matrice $e^{\mathbf{M} t}$. Pour cela il faut diagonaliser $\mathbf{M}=\mathbf{S D ~ S}^{-1}$ et on aura $e^{\mathbf{M} t}=\mathbf{S} e^{\mathrm{Dt}} \mathbf{S}^{-1}$. De manière générale la diagonalisation d'une matrice $8 \times 8$ implique la solution d'une équation du huitième degrée. Il est bien connu depuis les travaux de Galois et d'Abel que la solution n'est pas possible de façon analytique en termes de radicaux. Une approche géneraliste aura donc forcément recours à des solutions numériques. Cependant, la matrice $M$ peut être diagonalisée d'une manière très élégante en remarquant qu'elle possède la symétrie translationnelle. Nous essayerons ainsi de faire prévaloir la force d'une idée astucieuse sur une approche basée sur un calcul aveugle et laborieux. En effet, la topologie et plus précisément la connectivité de l'espace des configurations du problème est celle d'une chaîne linéaire aux conditions aux limites cycliques. Ce problème a une invariance translationnelle. La matrice $\mathbf{M}$ dans l'équation (9) peut s'écrire comme: $M_{i, j}=\delta_{i, j-1}-2 \delta_{i, j}+\delta_{i, j-1}$. (Dans cette expression il faut prendre les indices modulo 8). On voit que dans chaque point on a la même relation entre un point et ses premiers voisins. Une inspection de la situation locale ne permettra donc pas de dire dans quel site on est: c'est l'invariance translationnelle. Un problème de phonons avec huit atomes $\frac{d^{2}}{d t^{2}} \mathbf{U}=\frac{\kappa}{m} \mathbf{M U}$ fait intervenir la même matrice $M$. Les vecteurs propres peuvent donc s'écrire sous forme d'ondes de Bloch: 


$$
\mathbf{V}^{(q)}=\frac{1}{\sqrt{8}}\left(\begin{array}{c}
1 \\
e^{22 \pi q / 8} \\
e^{24 \pi q / 8} \\
e^{26 \pi q / 8} \\
e^{28 \pi q / 8} \\
e^{210 \pi q / 8} \\
e^{212 \pi q / 8} \\
e^{214 \pi q / 8}
\end{array}\right)
$$

oì $\left[\mathbf{V}^{(q)}\right]_{j}=\frac{1}{\sqrt{8}} e^{22 \pi(j-1) q / 8}$. Les valeurs propres associées sont $\lambda^{(q)}=\frac{4}{\tau} \sin ^{2}(\pi q / 8)$. Le théorème de Bloch ne fait qu'exprimer l'invariance translationnelle. Il s'ensuit pour la matrice colonne $\mathbf{P}(t)$ (dont la ligne $j$ donne la probabilité que le système prend la configuration $j$ à l'instant $t$ ) que:

$$
\mathbf{P}(t)=\mathbf{S} \boldsymbol{\Lambda} \mathbf{S}^{-1} \mathbf{P}(0)
$$

où

$$
\begin{aligned}
& \mathbf{S}=\frac{1}{\sqrt{8}}\left(\begin{array}{cccccccc}
1 & 1 & 1 & 1 & 1 & 1 & 1 & 1 \\
1 & e^{2 \pi / 4} & e^{2 \pi / 2} & e^{23 \pi / 4} & e^{2 \pi} & e^{25 \pi / 4} & e^{23 \pi / 2} & e^{27 \pi / 4} \\
1 & e^{2 \pi / 2} & e^{2 \pi} & e^{23 \pi / 2} & e^{22 \pi} & e^{25 \pi / 2} & e^{23 \pi} & e^{27 \pi / 2} \\
1 & e^{23 \pi / 4} & e^{23 \pi / 2} & e^{29 \pi / 4} & e^{23 \pi} & e^{215 \pi / 4} & e^{29 \pi / 2} & e^{221 \pi / 4} \\
1 & e^{2 \pi} & e^{22 \pi} & e^{23 \pi} & e^{24 \pi} & e^{25 \pi} & e^{26 \pi} & e^{27 \pi} \\
1 & e^{25 \pi / 4} & e^{25 \pi / 2} & e^{215 \pi / 4} & e^{25 \pi} & e^{25 \pi / 4} & e^{i 5 \pi / 2} & e^{235 \pi / 4} \\
1 & e^{23 \pi / 2} & e^{23 \pi} & e^{29 \pi / 2} & e^{26 \pi} & e^{215 \pi / 2} & e^{29 \pi} & e^{221 \pi / 2} \\
1 & e^{27 \pi / 4} & e^{27 \pi / 2} & e^{221 \pi / 4} & e^{27 \pi} & e^{235 \pi / 4} & e^{221 \pi / 2} & e^{249 \pi / 4}
\end{array}\right) \\
& \mathbf{S}^{-1}=\frac{1}{\sqrt{8}}\left(\begin{array}{cccccccc}
1 & 1 & 1 & 1 & 1 & 1 & 1 & 1 \\
1 & e^{-\imath \pi / 4} & e^{-\imath \pi / 2} & e^{-\imath 3 \pi / 4} & e^{-\imath \pi} & e^{-\imath 5 \pi / 4} & e^{-\imath 3 \pi / 2} & e^{-\imath 7 \pi / 4} \\
1 & e^{-\imath \pi / 2} & e^{-\imath \pi} & e^{-\imath 3 \pi / 2} & e^{-22 \pi} & e^{-\imath 5 \pi / 2} & e^{-\imath 3 \pi} & e^{-\imath 7 \pi / 2} \\
1 & e^{-\imath 3 \pi / 4} & e^{-\imath 3 \pi / 2} & e^{-\imath 9 \pi / 4} & e^{-\imath 3 \pi} & e^{-\imath 15 \pi / 4} & e^{-29 \pi / 2} & e^{-\imath 21 \pi / 4} \\
1 & e^{-\imath \pi} & e^{-22 \pi} & e^{-\imath 3 \pi} & e^{-\imath 4 \pi} & e^{-25 \pi} & e^{-\imath 6 \pi} & e^{-\imath 7 \pi} \\
1 & e^{-25 \pi / 4} & e^{-25 \pi / 2} & e^{-\imath 15 \pi / 4} & e^{-\imath 5 \pi} & e^{-\imath 25 \pi / 4} & e^{-\imath 15 \pi / 2} & e^{-\imath 35 \pi / 4} \\
1 & e^{-\imath 3 \pi / 2} & e^{-\imath 3 \pi} & e^{-\imath 9 \pi / 2} & e^{-26 \pi} & e^{-\imath 15 \pi / 2} & e^{-\imath 9 \pi} & e^{-\imath 21 \pi / 2} \\
1 & e^{-27 \pi / 4} & e^{-\imath 7 \pi / 2} & e^{-\imath 21 \pi / 4} & e^{-\imath 7 \pi} & e^{-\imath 35 \pi / 4} & e^{-\imath 21 \pi / 2} & e^{-\imath 49 \pi / 4}
\end{array}\right)
\end{aligned}
$$


et $\boldsymbol{\Lambda}$ est donné par:

$$
\left(\begin{array}{cccccccc}
1 & 0 & 0 & 0 & 0 & 0 & 0 & 0 \\
0 & e^{-\frac{4 t}{\tau} \sin ^{2}(\pi / 8)} & 0 & 0 & 0 & 0 & 0 & 0 \\
0 & 0 & e^{-\frac{4 t}{\tau} \sin ^{2}(\pi / 4)} & 0 & 0 & 0 & 0 & 0 \\
0 & 0 & 0 & e^{-\frac{4 t}{\tau} \sin ^{2}(3 \pi / 8)} & 0 & 0 & 0 & 0 \\
0 & 0 & 0 & 0 & e^{-\frac{4 t}{\tau} \sin ^{2}(\pi / 2)} & 0 & 0 & 0 \\
0 & 0 & 0 & 0 & 0 & e^{-\frac{4 t}{\tau} \sin ^{2}(5 \pi / 8)} & 0 & 0 \\
0 & 0 & 0 & 0 & 0 & 0 & e^{-\frac{4 t}{\tau} \sin ^{2}(3 \pi / 4)} & 0 \\
0 & 0 & 0 & 0 & 0 & 0 & 0 & e^{-\frac{4 t}{\tau} \sin ^{2}(7 \pi / 8)}
\end{array}\right)
$$

Ici $\boldsymbol{\Lambda}=e^{\mathbf{D} t}$ et $\mathbf{P}(0)$ contient les conditions initiales, c.à.d. les probabilités initiales de chaque configuration. Par exemple, si la configuration 1 est la configuration initiale nous devons prendre:

$$
\mathbf{P}^{(1)}(0)=\left(\begin{array}{l}
1 \\
0 \\
0 \\
0 \\
0 \\
0 \\
0 \\
0
\end{array}\right)
$$

De façon équivalente nous pouvons définir d'autres vecteurs "colonne" $\mathbf{P}^{(j)}(0)$. Nous considérons que les huit configurations initiales sont équiprobables à l'équilibre thermodynamique. Nous pouvons alors à l'aide de l'équation (12) faire le tableau de toutes les probabilités $p_{j k}(t)$ pour que le système se trouve dans la configuration $k$ à l'instant $t$ s'il se trouvait dans la configuration $j$ à l'instant 0 . Il suffit de juxtaposer les huit solutions (12) aux huit conditions initiales de type (14) pondérées chacune par un facteur $\frac{1}{8}$. Ce tableau s'écrit alors sous la forme:

$$
\left[\mathbf{P}^{(1)}(t), \mathbf{P}^{(2)}(t), \cdots, \mathbf{P}^{(8)}(t)\right]=\mathbf{S} \Lambda \mathbf{S}^{-1} \frac{1}{8} \amalg_{8 \times 8}
$$

où $\amalg_{8 \times 8}$ est la matrice unité de rang 8 résultant de la juxtaposition des huit vecteurs colonne $\mathbf{P}^{(j)}(0)$. Introduisons la matrice ligne $[\mathbb{F}(\boldsymbol{Q})]$ de type $1 \times 8$ qui contient les transformées de Fourier des huit configurations $\mathcal{C}_{i}$ : 


$$
[\mathbb{F}(\boldsymbol{Q})]=\left[\mathcal{F}_{\mathcal{C}_{1}}^{*}(\boldsymbol{Q}), \mathcal{F}_{\mathcal{C}_{2}}^{*}(\boldsymbol{Q}), \cdot s, \mathcal{F}_{\mathcal{C}_{8}}^{*}(\boldsymbol{Q})\right]
$$

Alors, la moyenne thermodynamique dans l'expression (7) peut s'écrire sous la forme:

$$
\left\langle\mathcal{F}^{*}(\boldsymbol{Q}, t) \mathcal{F}(\boldsymbol{Q}, 0)>_{t h}=\sum_{j, k} p_{j k}(t) \mathcal{F}_{\mathcal{C}_{j}}^{*}(\boldsymbol{Q}) \mathcal{F}_{\mathcal{C}_{k}}(\boldsymbol{Q})=\frac{1}{8}[\mathbb{F}(\boldsymbol{Q})] \mathbf{S} \boldsymbol{\Lambda} \mathbf{S}^{-1}[\mathbb{F}(\boldsymbol{Q})]^{\dagger}\right.
$$

Dans la configuration 1 , les sites occupés sont I, L et $O$, avec des vecteurs de position $\boldsymbol{e}_{1}, \boldsymbol{e}_{4}$ et $\boldsymbol{e}_{7}$. On peut vérifier que les sites occupés dans la configuration $j$ ont les vecteurs de position $e_{1 \oplus 3(j-1)}$, $e_{1 \oplus 3 j}$ et $e_{1 \oplus 3(j+1)}$, où le symbole $\oplus$ est supposé indiquer que l'on prend toujours le résultat modulo 8. Donc:

$$
\mathcal{F}_{\mathcal{C}_{j}}^{*}(\boldsymbol{Q})=e^{-\imath \boldsymbol{Q} \cdot \boldsymbol{e}_{1 \oplus 3(j-1)}}+e^{-\imath \boldsymbol{Q} \cdot \boldsymbol{e}_{1 \oplus 3 j}}+e^{-\imath \boldsymbol{Q} \cdot \boldsymbol{e}_{1 \oplus 3(j+1)}}
$$

Suivant la procédure décrite en (8), la fonction de diffusion totale n'est autre que la transformée de Fourier temporelle de l'expression (17). La transformée de Fourier temporelle ne concerne que $\Lambda$. Sa valeur $\mathcal{F}(\boldsymbol{\Lambda})(\omega)$ est donnée par:

$$
\left(\begin{array}{cccccccc}
\delta(\omega) & 0 & 0 & 0 & 0 & 0 & 0 & 0 \\
0 & \mathcal{L}\left(\Gamma_{1}, \omega\right) & 0 & 0 & 0 & 0 & 0 & 0 \\
0 & 0 & \mathcal{L}\left(\Gamma_{2}, \omega\right) & 0 & 0 & 0 & 0 & 0 \\
0 & 0 & 0 & \mathcal{L}\left(\Gamma_{3}, \omega\right) & 0 & 0 & 0 & 0 \\
0 & 0 & 0 & 0 & \mathcal{L}\left(\Gamma_{4}, \omega\right) & 0 & 0 & 0 \\
0 & 0 & 0 & 0 & 0 & \mathcal{L}\left(\Gamma_{5}, \omega\right) & 0 & 0 \\
0 & 0 & 0 & 0 & 0 & 0 & \mathcal{L}\left(\Gamma_{6}, \omega\right) & 0 \\
0 & 0 & 0 & 0 & 0 & 0 & 0 & \mathcal{L}\left(\Gamma_{7}, \omega\right)
\end{array}\right)
$$

où

$$
\begin{gathered}
\mathcal{L}(\gamma, \omega)=\frac{1}{\pi} \frac{\gamma}{\gamma^{2}+\omega^{2}} \\
\Gamma_{j}=\frac{4}{\tau} \sin ^{2}\left(\frac{\pi}{8} j\right)
\end{gathered}
$$




\section{$5 \quad$ Le calcul de la diffusion incohérente est plus compliqué}

Le calcul de l'expression (17) est laborieux. Nous n'en donnerons pas le résultat final, qui n'a d'ailleurs pas beaucoup d'intérêt. Actuellement, une seule équipe [9] a réussi à faire pousser un quasicristal octogonal. La quantité d'échantillon obtenue était très petite. Nous ne sommes donc pas encore en mesure de faire des études de sauts atomiques dans des quasicristaux octogonaux. Notre but n'était que d'illustrer une méthode pour en faire passer les idées conceptuelles. L'aspect abrutissant de la forêt de calculs explicites ne pourrait servir qu'à occulter ces idées. Nous devons faire quelques remarques:

- Dans l'equation (18) nous n'avons pas inclus les contributions des huit atomes immobiles en $\mathrm{A}, \mathrm{B}, \mathrm{C}, \mathrm{D}, \mathrm{E}, \mathrm{F}, \mathrm{G}, \mathrm{H}$. On peut démontrer que le fait de les inclure n'aurait pas modifié la partie quasiélastique de la réponse dynamique.

- Le pavage octogonal de Penrose possède la symétrie huit. Cependant dans chacune des huit configurations cette symétrie est localement brisée. L'espace des configurations nous fait retrouver la symétrie perdue et nous permet d'utiliser de façon générale la théorie des groupes. Ceci est illustré dans notre cas par l'utilisation du théorème de Bloch.

- Le même formalisme peut être utilisé pour calculer la fonction de diffusion incohérente. II faudra alors suivre également l'évolution de la position d'une particule marquée, à l'intérieur des configurations. Après un tour de huit sauts, la configuration de départ est retrouvée, mais la particule n'est plus au même endroit. Il faudra tourner encore deux fois pour récupérer la vraie situation de départ. Ceci nous emmène à considérer 24 configurations marquées. Etant donné que la position de la particule choisie doit être connue à l'intérieur de la configuration, le calcul de la fonction de diffusion incohérente est plus difficile que celui de la fonction de diffusion totale. Dans notre exemple, la matrice $24 \times 24$ peut de nouveau être diagonalisé en s'appuyant sur le théorème de Bloch.

- Enfin, le traitement, en termes de configurations, nous permet de décrire des corrélations complexes. Une approche en termes de sites relèverait certainement de la physique non-linéaire.

\section{Remarques finales}

Les calculs et leurs résultats sont donnés de façon explicite dans la référence [10]. Il nous reste à mentionner quelques autres exemples d'applications. Nous avons utilisé la même méthode pour le calcul des sauts atomiques dans un quasicristal icosaédrique. Le problème était le suivant. Sept atomes de cuivre sont repartis sur les vingt sommets d'un dodécaèdre. Il's ne peuvent pas être premiers voisins et ils ne peuvent pas occuper des sites opposés. Pour le reste, les atomes peuvent sauter entre des sites de premiers voisins si ces règles sont respectés. Le calcul a été fait dans [11]. Nous avons également utilisé cette méthode pour calculer les fonctions de diffusion rotationnelle 
d'une molécule de méthane partiellement deutériée $\mathrm{CD}_{3} \mathrm{H}$ ou $\mathrm{CDH}_{3}$. La brisure de la symétrie par la deutériation constituait une difficulté insurmontable pour les approches classiques basées sur la théorie des groupes. Nous avons vu comment cette symétrie est rétablie par l'introduction de l'espace des configurations. Les résultats de nos calculs pour le méthane deutérié sont accord avec une expérience effectuée par Michael Prager [12]. Ils sont résumés dans les tableaux suivants. Ces calculs feront l'objet d'une publication ultérieure [13].

Tableau 1. Fonctions de diffusion pour la rotation d'une molécule $\mathrm{CH}_{3} \mathrm{D}$.

\begin{tabular}{|l|c|}
\hline$S_{\text {inc }}(Q, \omega)$ & les longueurs de diffusion sont incohérentes \\
\hline$\delta(\omega)$ & $b_{C}^{2}+\frac{1}{4}\left(b_{D}^{2}+3 b_{H}^{2}\right)\left(1+3 j_{0}(2 Q \mathcal{A})\right)$ \\
\hline $\mathcal{L}\left(-\frac{8}{\sigma} ; \omega\right)$ & $\frac{1}{4}\left(3 b_{D}^{2}+b_{H}^{2}\right)\left(1-j_{0}(2 Q \mathcal{A})\right)$ \\
\hline $\mathcal{L}\left(-\frac{5}{\sigma}-\frac{3}{\tau} ; \omega\right)$ & $2 b_{H}^{2}\left(1-j_{0}(2 Q \mathcal{A})\right)$ \\
\hline
\end{tabular}

\begin{tabular}{|l|r|}
\hline$S(Q, \omega)$ & les longueurs de diffusion sont cohérentes \\
\hline$\delta(\omega)$ & $\begin{array}{r}b_{C}^{2}+\frac{1}{4}\left(b_{D}+3 b_{H}\right)^{2}\left(1+3 j_{0}(2 Q \mathcal{A})\right) \\
+2 b_{C}\left(b_{D}+3 b_{H}\right) j_{0}\left(Q \mathcal{A} \sqrt{\frac{3}{2}}\right)\end{array}$ \\
\hline $\mathcal{L}\left(-\frac{8}{\sigma}, \omega\right)$ & $\frac{3}{4}\left(b_{D}-b_{H}\right)^{2}\left(1-j_{0}(2 Q \mathcal{A})\right)$ \\
\hline
\end{tabular}




\section{References}

[1] L. Van Hove, Phys. Rev. 95, 249 (1954).

[2] M. Bée, Quasielastic Neutron Scattering, (Adam Hilger, Bristol, 1988). Voir aussi le cours de P. Damay dans le même volume.

[3] Ce jeu s'appelle "14-15 puzzle" ou "Boss puzzle" en Anglais. Dans la version originale du jeu, uniquement les pièces 14 et 15 étaient échangées et il fallait rétablir le bon ordre. Sam Lloyd (1841-1911) faisait miroiter une récompense importante pour la personne qui trouverait une solution de ce problème. Cette solution n'existe pas: seulement les permutations paires sont permises. Le jeu était très populaire aux Etats-Unis dans les années 1870. Voir M. Garder, Scientific Am., August 1957, pp.120-127.

[4] R.P. Feynman, The Feynman Lectures of Physics, Quantum Mechan$i c s$, R.P. Feynman, R.B. Leighton and M. Sands eds., (Addison-Wesley, Reading, Massachusetts, 1963).

[5] A. Tomonura, J. Endo, T. Matsuda and T. Kawasaki, Am. J. Phys. 57 (1989) 117. Voir également M.P. Silverman dans More than one Mystery, Explorations in Quantum Interference, (Springer, New York, 1995).

[6] Par notre formulation nous voulons faire resortir que certaines particules identiques ne peuvent être échangées, par exemple deux protons de deux groupes $\mathrm{CH}_{3}$ différents.

[7] Dans le jeu de pousse-pousse il existe une bijection entre la particule abstraite et la position du trou. Le problème est donc isomorphe à celui de la diffusion d'une lacune par sauts entre sites premiers voisins.

[8] P.A. Kalugin and A. Katz, Europhys. Lett. 21, 921 (1993).

[9] N. Wang, H. Chen and K.H. Kuo, Phys. Rev. Lett. 59, 1010 (1987).

[10] G. Coddens, J. Phys. I France 4, 921 (1994).

[11] G. Coddens and S. Lyonnard, Physica B 226, 28 (1996); 233, 93 (1997).

[12] M. Prager, communication personnelle.

[13] G. Coddens, en préparation. 